\title{
DOMINATION AND EDGE DOMINATION IN TREES ${ }^{1}$
}

\author{
B. Senthilkumar ${ }^{\dagger}$, Y. B. Venkatakrishnan ${ }^{\dagger \dagger}$, H. Naresh Kumar ${ }^{\dagger \dagger \dagger}$ \\ SASTRA Deemed University, Tanjore, Tamilnadu, India \\ ${ }^{\dagger}$ senthilsubramanyan@gmail.com ††venkatakrish2@maths.sastra.edu \\ ††'nareshhari1403@gmail.com
}

\begin{abstract}
Let $G=(V, E)$ be a simple graph. A set $S \subseteq V$ is a dominating set if every vertex in $V \backslash S$ is adjacent to a vertex in $S$. The domination number of a graph $G$, denoted by $\gamma(G)$ is the minimum cardinality of a dominating set of $G$. A set $D \subseteq E$ is an edge dominating set if every edge in $E \backslash D$ is adjacent to an edge in $D$. The edge domination number of a graph $G$, denoted by $\gamma^{\prime}(G)$ is the minimum cardinality of an edge dominating set of $G$. We characterize trees with domination number equal to twice edge domination number.

Keywords: Edge dominating set, Dominating set, Trees.
\end{abstract}

\section{Introduction}

Domination theory is a well studied topic in graph theory. Depending on the utility in real life application, domination on vertex set and on edge set has been defined. Edge dominating set is used to study the behaviour of telephone switching network [5] built to phone calls from one telephone to another telephone at a time. Edge dominating set is also used in deterministic distributed algorithms in networks with unique node identifier in port numbered network. Dominating set is used to identify the minimum number of servers in an adhoc network. For different dominating parameters, the reader is refered to two excellent books $[2,3]$.

In domination theory, comparison is made between domination parameters defined on vertex set or domination parameters defined on edge set. There are only a few studies on comparison between domination parameter defined on vertex set with a domination parameter defined on edge set, see $[4,8]$. Here, a domination parameter defined on edge set, edge domination, is compared with a domination parameter defined on vertex set, vertex domination and we characterize trees with domination number equal to twice edge domination number.

Let $G=(V, E)$ be a simple connected graph. Two edges are adjacent if they are incident with a common vertex. Two vertices $u$ and $v$ are adjacent if there is an edge $e$ incident with both $u$ and $v$. For every vertex $v \in V$, the set of all vertices adjacent to $v$ is an open neighborhood of the vertex $v$ denoted by $N(v)$ and the set $N[v]=N(v) \cup\{v\}$ is called the closed neighborhood of vertex $v$. The degree of a vertex $v$ is the cardinality of its open neighborhood, denoted $d_{G}(v)=|N(v)|$. A vertex of degree one is called a leaf and its neighbor is called a support vertex. A support vertex with more than one leaf is called a strong support vertex and a support vertex with exactly one leaf is called a weak support vertex. The number of edges between $u$ and $v$ in a shortest path is the distance between vertices $u$ and $v$. The longest distance between any pair of vertices is defined as the diameter of the graph $G$, and is denoted by $\operatorname{diam}(G)$. A path on $n$ vertices is denoted by $P_{n}$. A vertex $v$ in a tree $T$ is adjacent to a path $P_{n}$ through its vertex $x$, if a path containing $x$ is one of the components of $(T-v x)$. A star of order $n \geq 2$, denoted by $K_{1, n-1}$, is a tree with at least $(n-1)$ leaves. A double star is a tree with exactly two support vertices and is denoted by $D_{r, s}$, where $r$ and $s$ are the number of leaves attached to each support vertices.

\footnotetext{
${ }^{1}$ This work is supported by TATA-Realty and Infrastructure Limited.
} 
A subset $S$ of $V$ is a dominating set abbreviated DS, if every vertex in $V \backslash S$ is adjacent to some vertex in $S$. The domination number, $\gamma(G)$ of a graph $G$, is the minimum cardinality of a DS of $G$. By $\gamma(G)$-set, we mean a DS with minimum cardinality of a graph $G$.

A subset $D$ of $E$ is a edge dominating set abbreviated EDS, if every edge in $E \backslash D$ is adjacent to some edge in $D$. The edge domination number, $\gamma^{\prime}(G)$ of a graph $G$, is the minimum cardinality of a EDS of $G$. By $\gamma^{\prime}(G)$-set, we mean an EDS with minimum cardinality of graph $G$. For more properties on edge dominating set, refer the reader to $[1,7]$.

Characterizing trees with equal dominating parameters is available in the literature, see [6]. We characterize trees with domination number equal to twice edge domination number.

\section{Main results}

We begin this section with a theorem.

Theorem 1. For any tree $T, \gamma^{\prime}(T) \leq \gamma(T) \leq 2 \gamma^{\prime}(T)$.

P r o o f. Let $D$ be a $\gamma^{\prime}(T)$-set. Let $S$ be the set of vertices incident with the edges of $D$. The set $S$ is a DS of tree $T$. Thus $\gamma(T) \leq|S| \leq 2|D|=2 \gamma^{\prime}(T)$.

Let $S$ be a $\gamma(T)$-set. For each vertex of $S$, select exactly one edge incident with it, and call such a set of edges as $D$. Then $D$ is an EDS of tree $T$. We have $\gamma^{\prime}(T) \leq|D|=|S|=\gamma(T)$.

For the purpose of characterizing trees with equal domination number and twice edge domination number, we introduce the family $\mathcal{A}$ of trees $T=T_{k}$ that can be obtained as follows.

Let $T_{1}=P_{4}$. If $k \geq 2$, then $T_{k+1}$ can be obtained recursively from $T_{k}$ by one of the following operations:

- Operation $\mathcal{O}_{1}$ : Attach a vertex to a support vertex of $T_{k}$.

- Operation $\mathcal{O}_{2}$ : Attach a 4-path by joining its support vertex to a vertex of $T_{k}$ adjacent to a 4-path through its support vertex.

- Operation $\mathcal{O}_{3}$ : Attach a 4-path by joining its support vertex to a support vertex of $T_{k}$.

- Operation $\mathcal{O}_{4}$ : Attach a double star $D_{r, s}$ with $r \cdot s \geq 2$ by joining a leaf adjacent to a strong support vertex to a vertex of $T_{k}$ adjacent to a 2-path.

- Operation $\mathcal{O}_{5}$ : Attach a double star $D_{r, s}$ with $r \cdot s \geq 2$ by joining a leaf adjacent to a strong support vertex to a support vertex of $T_{k}$.

The operations given above are illustrated in Figure 1. It is proved that $\gamma(T)=2 \gamma^{\prime}(T)$ for every tree $T$ of the family $\mathcal{A}$.

Lemma 1. If $T \in \mathcal{A}$, then $\gamma(T)=2 \gamma^{\prime}(T)$.

P r o o f. To construct the tree $T$, we use the method of induction on the number $k$ of operations. If $T=P_{4}$, then obviously $\gamma(T)=2=2 \gamma^{\prime}(T)$. Let $k$ be a positive integer. Assume that the result is true for every $T^{\prime}=T_{k}$ of the family $\mathcal{A}$ constructed by $k-1$ operations. Let $T=T_{k+1}$ be a tree of the family $\mathcal{A}$ constructed by $k$ operations.

First assume that $T$ is obtained from $T^{\prime}$ by operation $\mathcal{O}_{1}$. Let $D^{\prime}$ be a $\gamma^{\prime}\left(T^{\prime}\right)$-set. It is easy to see that $D^{\prime}$ is an EDS of tree $T$. Thus $\gamma^{\prime}(T) \leq \gamma^{\prime}\left(T^{\prime}\right)$. Obviously, $\gamma\left(T^{\prime}\right) \leq \gamma(T)$. We now get 

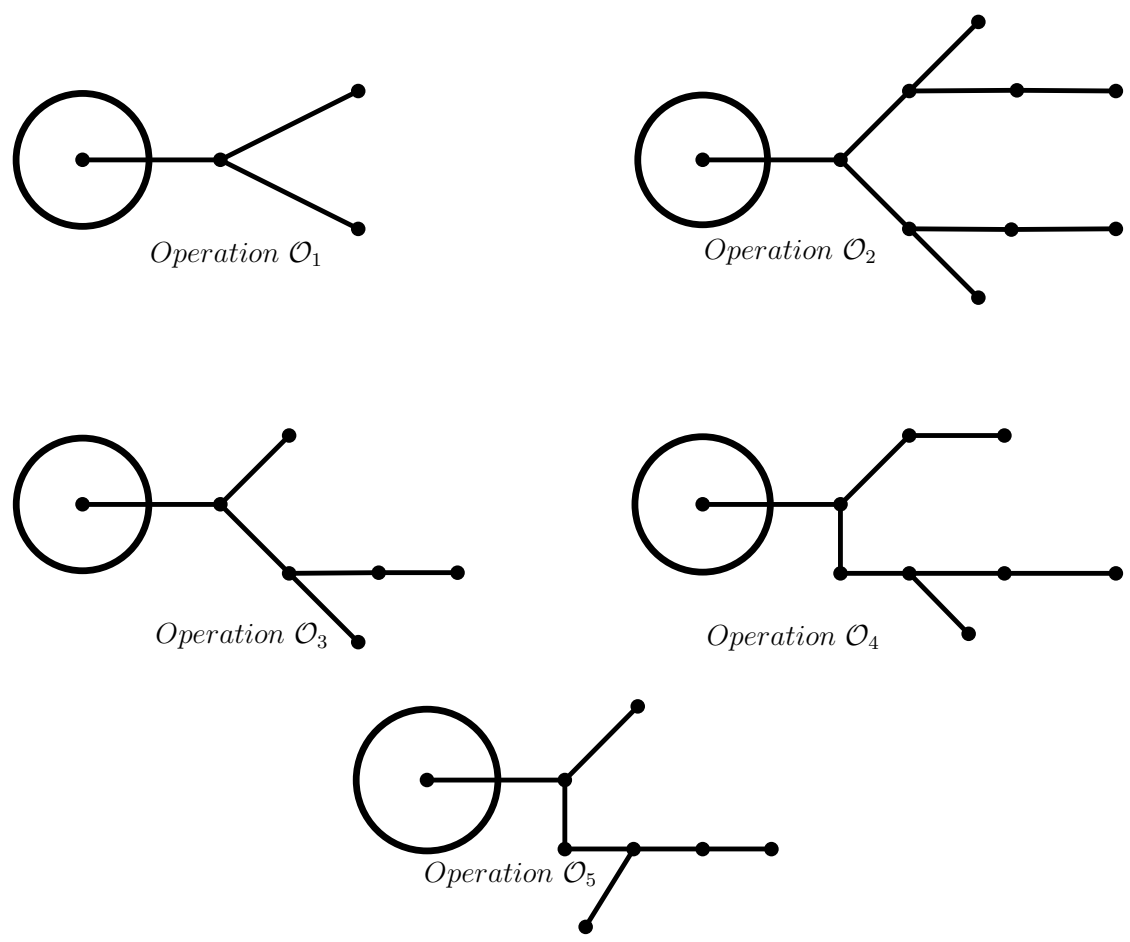

Figure 1. Operations $\mathcal{O}_{1}$ to $\mathcal{O}_{5}$

$2 \gamma^{\prime}(T) \leq 2 \gamma^{\prime}\left(T^{\prime}\right)=\gamma\left(T^{\prime}\right) \leq \gamma(T)$. On the other hand by Theorem 1 , we have $\gamma(T) \leq 2 \gamma^{\prime}(T)$. This implies that $\gamma(T)=2 \gamma^{\prime}(T)$.

Now assume that the tree $T$ is obtained from the tree $T^{\prime}$ by the operation $\mathcal{O}_{2}$. Let $x$ be the vertex to which a 4-path pqrs is joined through $q$. Let $q$ be adjacent to $x$. Let abcd be a path different from path pqrs with the vertex $b$ adjacent to $x$. Let $D^{\prime}$ be a $\gamma^{\prime}(T)$-set. To dominate the edges $c d, b c, a b$ and $b x$, the edge $b c \in D^{\prime}$. It is clear that $D^{\prime} \cup\{q r\}$ is an EDS of $T$. Thus $\gamma^{\prime}(T) \leq \gamma^{\prime}\left(T^{\prime}\right)+1$. Let $S$ be a $\gamma(T)$-set. To dominate the vertices $d, a, s$ and $p$, the vertices $c, b, r, q \in S$. It is obvious that $S \backslash\{q, r\}$ is a DS of tree $T^{\prime}$. Thus $\gamma\left(T^{\prime}\right) \leq \gamma(T)-2$. We obtain $2 \gamma^{\prime}(T) \leq 2 \gamma^{\prime}\left(T^{\prime}\right)+2=\gamma\left(T^{\prime}\right)+2 \leq \gamma(T)$. We conclude that $2 \gamma^{\prime}(T)=\gamma(T)$.

Now assume that $T$ is obtained from $T^{\prime}$ by the operation $\mathcal{O}_{3}$. Let $x$ be the vertex to which the 4-path pqrs is attached by joining $q$ and $x$. Let $y$ be a leaf adjacent to $x$. Let $D^{\prime}$ denote a $\gamma^{\prime}\left(T^{\prime}\right)$-set. It is clear that $D^{\prime} \cup\{q, r\}$ is an EDS of tree $T$. Thus $\gamma^{\prime}(T) \leq \gamma^{\prime}\left(T^{\prime}\right)+1$. Let $S$ be a $\gamma(T)$-set. To dominate the vertices $s, p$ and $y$, the vertices $r, q, x \in S$. It is obvious that $S \backslash\{q, r\}$ is a dominating set of the tree $T^{\prime}$. Thus $\gamma\left(T^{\prime}\right) \leq \gamma(T)-2$. We now get $2 \gamma^{\prime}(T) \leq 2 \gamma^{\prime}\left(T^{\prime}\right)+2=\gamma\left(T^{\prime}\right)+2 \leq \gamma(T)$. We conclude that $2 \gamma^{\prime}(T)=\gamma(T)$.

Now assume that $T$ is obtained from $T^{\prime}$ by the operation $\mathcal{O}_{4}$. Let $p$ and $q$ be support vertices of a double star. Let $r$ and $s$ be two leaves adjacent to $p$, and $t$ be the leaf adjacent to $q$. Denote by $x$ the vertex to which the double star is attached. Let $r$ be adjacent to $x$. Let $x$ be adjacent to 2-path $a b$ with $a$ adjacent to $x$. Let $D^{\prime}$ be a $\gamma\left(T^{\prime}\right)$-set. To dominate the edge $a b$, the edge $x a \in D^{\prime}$. The set $D^{\prime} \cup\{p q\}$ is an EDS of tree $T$. Thus $\gamma^{\prime}(T) \leq \gamma^{\prime}\left(T^{\prime}\right)+1$. Let $S$ be a $\gamma(T)$-set. To dominate the vertices $t, s$ and $b$, the vertices $q, p, a \in S$. It is easy to observe $S \backslash\{p, q\}$ is a dominating set of the tree $T^{\prime}$. Thus $\gamma\left(T^{\prime}\right) \leq \gamma(T)-2$. We now get $2 \gamma^{\prime}(T) \leq 2 \gamma^{\prime}\left(T^{\prime}\right)+2=\gamma\left(T^{\prime}\right)+2 \leq \gamma(T)$. We conclude that $\gamma(T)=2 \gamma^{\prime}(T)$.

Assume that $T$ is obtained from $T^{\prime}$ by the operation $\mathcal{O}_{5}$. Let $p$ and $q$ be support vertices of 
the attached double star. Let $r$ and $s$ be two leaves adjacent to $p$, and $t$ be a leaf adjacent to $q$. Denote by $x$ the support vertex to which the double star is attached. Let $r$ be adjacent to $x$. The leaf adjacent to $x$ is denoted by $y$. Let $D^{\prime}$ represent a $\gamma^{\prime}\left(T^{\prime}\right)$-set. The set $D^{\prime} \cup\{p q\}$ is an EDS of the tree $T$. Thus $\gamma^{\prime}(T) \leq \gamma^{\prime}\left(T^{\prime}\right)+1$. Let $S$ be a $\gamma(T)$-set. The vertices $t, s$ and $y$, are dominated by the vertices $p, q, x \in S$. The set $S \backslash\{p, q\}$ is a DS of the tree $T^{\prime}$. Thus $\gamma\left(T^{\prime}\right) \leq \gamma(T)-2$. We now get $2 \gamma^{\prime}(T) \leq 2 \gamma^{\prime}\left(T^{\prime}\right)+2=\gamma\left(T^{\prime}\right)+2 \leq \gamma(T)$. We conclude that $\gamma(T)=2 \gamma^{\prime}(T)$.

We now prove that if $2 \gamma^{\prime}(T)=\gamma(T)$, then the tree belongs to the family $\mathcal{A}$.

Lemma 2. Let $T$ be a tree. If $2 \gamma^{\prime}(T)=\gamma(T)$, then $T \in \mathcal{A}$.

P r o o f. If $\operatorname{diam}(T)=1$, then $T=P_{2}$. We have $\gamma\left(P_{2}\right)=1<2=2 \gamma^{\prime}\left(P_{2}\right)$. If $\operatorname{diam}(T)=2$, then $T$ is a star. We have $\gamma\left(P_{3}\right)=1<2=2 \gamma^{\prime}\left(P_{3}\right)$. If $\operatorname{diam}(T)=3$, the tree $T$ is a double star. If $T=P_{4}$, then $T \in \mathcal{A}$. If $T$ is a double star other than $P_{4}$, then $T$ is obtained from $P_{4}$ by required number of operations $\mathcal{O}_{1}$. Thus $T \in \mathcal{A}$. Let $\operatorname{diam}(T) \geq 4$. Thus the order $n$ of the tree $T$ is at least five. The method of induction on the order $n$ is used to prove the result. Assume that the lemma is valid for every tree $T^{\prime}$ of order $n^{\prime}<n$.

Assume that the support vertex of $T$, say $x$, is strong. Let $p$ and $q$ be leaves adjacent to $x$. Let $T^{\prime}=T-p$. Let $D$ be a $\gamma^{\prime}(T)$-set. If $x p \in D$ then $(D \backslash\{x p\}) \cup\{x q\}$ is an EDS of $T^{\prime}$. If $x p \notin D$ then obviously $D$ is an EDS of $T^{\prime}$. Thus $\gamma^{\prime}\left(T^{\prime}\right) \leq \gamma^{\prime}(T)$. Let $S^{\prime}$ be a $\gamma\left(T^{\prime}\right)$-set. Obviously $S^{\prime}$ is a DS of the tree $T$. Thus $\gamma(T) \leq \gamma\left(T^{\prime}\right)$. We now get $2 \gamma^{\prime}\left(T^{\prime}\right) \leq 2 \gamma^{\prime}(T)=\gamma(T) \leq \gamma\left(T^{\prime}\right)$. This implies that $\gamma\left(T^{\prime}\right)=2 \gamma^{\prime}\left(T^{\prime}\right)$. We have $T^{\prime} \in \mathcal{A}$ from the inductive hypothesis. The tree $T$ is obtained from $T^{\prime}$ by operation $\mathcal{O}_{1}$. Thus $T \in \mathcal{A}$. Hereafter, it is assumed that every support vertex of $T$ is weak.

Let $r$ be a vertex of maximum eccentricity diam $(T)$. We assume that $r$ is the root of the tree $T$. The leaf at a maximum distance from $r$ is denoted by $t, t$ be the child of $v$, let $v$ be the child of $u$ in the rooted tree. If $\operatorname{diam}(T) \geq 4$, then let $u$ be the child of $w$. If $\operatorname{diam}(T) \geq 5$, then let $w$ be the child of $d$. If $\operatorname{diam}(T) \geq 6$, then let $d$ be the child of $e$. The subtree induced by descendants of $x$ and a vertex $x$ in the rooted tree $T$ is denoted by $T_{x}$.

Among the children of $u$ assume that there is a support vertex, say $x$, other than $v$. Let $y$ be the leaf adjacent to $x$. Let $T^{\prime}=T-T_{v}$. Let $D^{\prime}$ be a $\gamma\left(T^{\prime}\right)$-set. The set $D^{\prime} \cup\{v\}$ is a DS of $T$. Thus $\gamma(T) \leq \gamma\left(T^{\prime}\right)+1$. Let $S$ be a $\gamma^{\prime}(T)$-set. To dominate the edges $v t$ and $x y$, the edges $u v, u x \in S$. It is obvious that $S \backslash\{u v\}$ is EDS of the tree $T^{\prime}$. Thus $\gamma^{\prime}\left(T^{\prime}\right) \leq \gamma^{\prime}(T)-1$. We obtain

$$
2 \gamma^{\prime}\left(T^{\prime}\right) \leq 2 \gamma^{\prime}(T)-2=\gamma(T)-2 \leq \gamma\left(T^{\prime}\right)+1-2<\gamma\left(T^{\prime}\right) .
$$

By Theorem 1 this case is impossible.

Assume that some child of $u$, say $x$, is a leaf. By the choice of diametrical path, the vertex $w$ is adjacent to isomorphic copy of $T_{u}$ or adjacent to path $P_{3}$ or adjacent to path $P_{2}$ or a support vertex of $T$.

Case (i): Let $w$ be adjacent to isomorphic copy of $T_{u}$, say $T_{u^{\prime}}$. Let $T_{u^{\prime}}=t^{\prime} v^{\prime} u^{\prime} x^{\prime}$. Let $u^{\prime}$ be adjacent to $w$. Let $T^{\prime}=T-T_{u}$. Let $D$ be a $\gamma^{\prime}(T)$-set. To dominate the edges $v t, u v, u x, v^{\prime} t^{\prime}, u^{\prime} v^{\prime}$ and $u^{\prime} x^{\prime}$, the edges $u v, u^{\prime} v^{\prime} \in D$. It is obvious that $D \backslash\{u v\}$ is an EDS of $T^{\prime}$. Thus $\gamma^{\prime}\left(T^{\prime}\right) \leq \gamma^{\prime}(T)-1$. Let $S^{\prime}$ be a $\gamma\left(T^{\prime}\right)$-set. The set $S^{\prime} \cup\{u, v\}$ is a DS of the tree $T$. Thus $\gamma(T) \leq \gamma\left(T^{\prime}\right)+2$. We now obtain $2 \gamma^{\prime}\left(T^{\prime}\right) \leq 2 \gamma^{\prime}(T)-2=\gamma(T)-2 \leq \gamma\left(T^{\prime}\right)$. This gives that $\gamma\left(T^{\prime}\right)=2 \gamma^{\prime}\left(T^{\prime}\right)$. The tree $T^{\prime} \in \mathcal{A}$ by the inductive hypothesis. The tree $T$ can be constructed from $T^{\prime}$ by operation $\mathcal{O}_{2}$. Thus $T \in \mathcal{A}$.

Case (ii): Let $w$ be adjacent to a 3-path $a b c$. Let $a$ be adjacent to $w$. Let $T^{\prime}=T-T_{a}$. Let $D$ be a $\gamma^{\prime}(T)$-set. To dominate the edges $v t, u v, u x, u w, w a, a b$ and $b c$, the edges $u v, a b \in D$. It is clear that $D \backslash\{a b\}$ is an EDS of the tree $T^{\prime}$. Thus $\gamma^{\prime}\left(T^{\prime}\right) \leq \gamma^{\prime}(T)-1$. Let $S^{\prime}$ be a $\gamma\left(T^{\prime}\right)$-set. The set $S^{\prime} \cup\{b\}$ is obviously a DS of tree $T$. Thus $\gamma(T) \leq \gamma\left(T^{\prime}\right)+1$. We now obtain

$$
2 \gamma^{\prime}\left(T^{\prime}\right) \leq 2 \gamma^{\prime}(T)-2=\gamma(T)-2 \leq \gamma\left(T^{\prime}\right)+1-2<\gamma\left(T^{\prime}\right) .
$$


By Theorem 1 this case is impossible.

Case (iii): Suppose $w$ is adjacent to 2-path $x y$. Let $w$ be adjacent to 2-path $x^{\prime} y^{\prime}$ different from 2-path $x y$. Let $T^{\prime}=T-T_{x}$. Let $D$ be a $\gamma^{\prime}(T)$-set. To dominate the edges $w x, x y, w x^{\prime}$ and $x^{\prime} y^{\prime}$, the edges $w x, w x^{\prime} \in D$. The set $D \backslash\{w x\}$ is an EDS of $T^{\prime}$. Thus $\gamma^{\prime}\left(T^{\prime}\right) \leq \gamma^{\prime}(T)-1$. Let $S^{\prime}$ be a $\gamma\left(T^{\prime}\right)$-set. The set $S^{\prime} \cup\{x\}$ is clearly a DS of tree $T$. Thus $\gamma(T) \leq \gamma\left(T^{\prime}\right)+1$. We now obtain $2 \gamma^{\prime}\left(T^{\prime}\right) \leq 2 \gamma^{\prime}(T)-2=\gamma(T)-2 \leq \gamma\left(T^{\prime}\right)+1-2<\gamma\left(T^{\prime}\right)$. Suppose the vertex $w$ is adjacent to exactly one 2-path $x y$. Let $T^{\prime}=T-T_{w}$. Let $D$ be a $\gamma^{\prime}(T)$-set. The edges $u v$ and $w x$ are in $D$. The set $D \backslash\{u v, w x\}$ is an EDS of tree $T^{\prime}$. Thus $\gamma^{\prime}\left(T^{\prime}\right) \leq \gamma^{\prime}(T)-2$. Let $S^{\prime}$ be a $\gamma\left(T^{\prime}\right)$-set. It is obvious that $S^{\prime} \cup\{u, v, x\}$ is a DS of tree $T$. Thus $\gamma(T) \leq \gamma\left(T^{\prime}\right)+3$. We now get

$$
2 \gamma^{\prime}\left(T^{\prime}\right) \leq 2 \gamma^{\prime}(T)-4=\gamma(T)-4 \leq \gamma\left(T^{\prime}\right)+3-4<\gamma\left(T^{\prime}\right) .
$$

By Theorem 1 this case is impossible.

Case (iv): The vertex $w$ is a support vertex. Let $y$ be the leaf adjacent to $w$. Let $T^{\prime}=T-T_{u}$. Let $D$ be a $\gamma^{\prime}\left(T^{\prime}\right)$-set. To dominate the edges $v t, u x$ and $w y$, the edges $u v, e \in D$ where $e$ is the edge incident with $w$ other than $w y$. It is obvious that $D \backslash\{u v\}$ is an EDS of tree $T^{\prime}$. Thus $\gamma^{\prime}\left(T^{\prime}\right) \leq \gamma^{\prime}(T)-1$. Let $S^{\prime}$ be a $\gamma\left(T^{\prime}\right)$-set. The set $S^{\prime} \cup\{u, v\}$ is obviously a DS of tree $T$. This gives $\gamma(T) \leq \gamma\left(T^{\prime}\right)+2$. We now get $2 \gamma^{\prime}\left(T^{\prime}\right) \leq 2 \gamma^{\prime}(T)-2=\gamma(T)-2 \leq \gamma\left(T^{\prime}\right)$. This implies that $2 \gamma^{\prime}\left(T^{\prime}\right)=\gamma\left(T^{\prime}\right)$. The tree $T^{\prime} \in \mathcal{A}$ by the inductive hypothesis. The tree $T$ can be constructed from $T^{\prime}$ by operation $\mathcal{O}_{3}$. Thus $T \in \mathcal{A}$.

Case (v): Now assume $d_{T}(w)=2$. By the choice of the diametrical path, the vertex $d$ is adjacent to isomorphic copy of $T_{w}$ or path $P_{4}$ or path $P_{3}$ or path $P_{2}$ or $w$ is a support vertex or $d_{T}(d)=2$.

Subcase (i): The vertex $d$ is adjacent to isomorphic copy of $T_{w}$. Let $D$ be a $\gamma^{\prime}(T)$-set. To dominate the edges $v t, u v, u x$ and $u w$ the edge $u v \in D$. To dominate the edges in the isomorphic copy, the edges $u^{\prime} v^{\prime} \in D$. To dominate the edges incident with $d$, the edge de $\in D$. Let $S$ be the set of vertices incident with edges in $D$. Clearly $|S| \leq 2|D|$. The set $S \backslash\{d\}$ is a DS of $T$. We have $\gamma(T) \leq 2|D|-1=2 \gamma^{\prime}(T)-1<2 \gamma^{\prime}(T)$.

Subcase (ii): The vertex $d$ is adjacent to 4-path $a^{\prime} b^{\prime} c^{\prime} d^{\prime}$ with $a^{\prime}$ adjacent to $d$. Let $D$ be a $\gamma^{\prime}(T)$-set. As in subcase (i), the edge $u v \in D$. To dominate the edge $d a^{\prime}$ and the edges in path $P_{4}: a^{\prime} b^{\prime} c^{\prime} d^{\prime}$, the edges $d e, b^{\prime} c^{\prime} \in D$. Let $S$ be the set of vertices incident with edges in $D$. Clearly $|S| \leq 2|D|$. The set $S \backslash\left\{b^{\prime}\right\}$ is a DS of $T$. We have $\gamma(T) \leq 2|D|-1=2 \gamma^{\prime}(T)-1<2 \gamma^{\prime}(T)$.

Subcase (iii): The vertex $d$ is adjacent to 3-path $a^{\prime} b^{\prime} c^{\prime}$ with $a^{\prime}$ adjacent to $d$. Let $D$ be a $\gamma^{\prime}(T)$-set. As in subcase (i), the edge $u v \in D$. To dominate the edge $b^{\prime} c^{\prime}$, the edge $a^{\prime} b^{\prime} \in D$. Let $S$ be the set of vertices incident with edges in $D$. Clearly $|S| \leq 2|D|$. The set $S \backslash\left\{a^{\prime}\right\}$ is a DS of $T$. We have $\gamma(T) \leq 2|D|-1=2 \gamma^{\prime}(T)-1<2 \gamma^{\prime}(T)$.

Subcase (iv): The vertex $d$ is adjacent to a 2-path $a^{\prime} b^{\prime}$ with $a^{\prime}$ adjacent to $d$. Let $T^{\prime}=T-T_{w}$. Let $D$ be a $\gamma^{\prime}(T)$-set. As in subcase (i), the edge $u v \in D$. To dominate the edges $d w$ and $a^{\prime} b^{\prime}$, the edge $d a^{\prime} \in D$. It is easy to observe that $D \backslash\{u v\}$ is an EDS of $T^{\prime}$. Thus $\gamma^{\prime}\left(T^{\prime}\right) \leq \gamma^{\prime}(T)-1$. Let $S^{\prime}$ be a $\gamma\left(T^{\prime}\right)$-set. The set $S^{\prime} \cup\{u, v\}$ is easily seen to be a DS of the tree $T$. Thus $\gamma(T) \leq \gamma\left(T^{\prime}\right)+2$. We now obtain $2 \gamma^{\prime}\left(T^{\prime}\right) \leq 2 \gamma^{\prime}(T)-2=\gamma(T)-2 \leq \gamma\left(T^{\prime}\right)$. This gives that $2 \gamma^{\prime}\left(T^{\prime}\right)=\gamma\left(T^{\prime}\right)$. The tree $T^{\prime} \in \mathcal{A}$ by the inductive hypothesis. The tree $T$ can be constructed from $T^{\prime}$ by operation $\mathcal{O}_{4}$. Thus $T \in \mathcal{A}$.

Subcase (v): The vertex $d$ is a support vertex. Let $y$ be the leaf adjacent to $d$. Let $T^{\prime}=T-T_{w}$. Arguing as in the previous subcase, we get $\gamma\left(T^{\prime}\right)=2 \gamma^{\prime}\left(T^{\prime}\right)$. The tree $T^{\prime} \in \mathcal{A}$ by the inductive hypothesis. The tree $T$ can be constructed from $T^{\prime}$ by operation $\mathcal{O}_{5}$. Thus $T \in \mathcal{A}$.

Subcase (vi): Now assume the degree of the vertex $d$ is two. Let $D$ be a $\gamma^{\prime}(T)$-set. To dominate the edges in $T_{w}$, the edge $u v \in D$. To dominate the edge $d w$, the edge $d e \in D$. Let $S$ 
be the set of vertices incident with edges of $D$. Clearly $|S| \leq 2|D|$. The set $S \backslash\{d\}$ is a DS of the tree $T$. Thus $\gamma(T) \leq 2|D|-1=2 \gamma^{\prime}(T)-1<2 \gamma^{\prime}(T)$.

Now assume $d_{T}(u)=2$. Let $T^{\prime}=T-T_{u}$. Let $D$ be a $\gamma^{\prime}(T)$-set. To dominate the edge $v t$, the edge $u v \in D$. The set $D \backslash\{u v\}$ is verified to be an EDS of tree $T^{\prime}$. Thus $\gamma^{\prime}\left(T^{\prime}\right) \leq \gamma^{\prime}(T)-1$. Let $S^{\prime}$ be a $\gamma\left(T^{\prime}\right)$-set. The set $S^{\prime} \cup\{v\}$ is obviously a DS of tree $T$. Thus $\gamma(T) \leq \gamma\left(T^{\prime}\right)+1$. We now obtain $2 \gamma^{\prime}\left(T^{\prime}\right) \leq 2 \gamma^{\prime}(T)-2=\gamma(T)-2 \leq \gamma\left(T^{\prime}\right)+1-2<\gamma\left(T^{\prime}\right)$. By Theorem 1 this case is impossible.

Characterization of trees with equal domination and twice the edge domination number is an immediate consequence of Lemma 1 and 2 and is stated as a theorem below.

Theorem 2. Let $T$ be a tree. Then $2 \gamma^{\prime}(T)=\gamma(T)$ if and only if $T \in \mathcal{A}$.

\section{Concluding remarks}

In this paper we characterize trees with domination number equal to twice edge domination number and present some problems for further research, among them we note the following:

1. Characterize graphs with equal domination number and twice edge domination number.

2. Characterize trees with equal domination number and edge domination number.

3. Characterize graphs with equal domination number and edge domination number.

\section{Acknowledgements}

The authors thank the anonymous referees for their helpful and constructive comments leading to improvements in the presentation of the paper.

\section{REFERENCES}

1. Arumugam S., Velammal S. Edge domination in graphs. Taiwanese J. Math., 1998. Vol. 2, No. 2. P. 173179. URL: https://www.jstor.org/stable/43834391

2. Haynes T. W., Hedetniemi S. T., Slater P. J. Fundamentals of Domination in Graphs. New York: Marcel Dekker, 1998. 455 p.

3. Haynes T. W., Hedetniemi S. T., Slater P. J. Domination in Graphs: Advanced Topics. New York: Marcel Dekker, 1998. 497 p.

4. Krishnakumari B., Venkatakrishnan Y.B., Krzywkowski M. On trees with total domination number equal to edge-vertex domination number plus one. Proc. Math. Sci., 2016. Vol. 126. P. 153-157. DOI: $10.1007 / \mathrm{s} 12044-016-0267-6$

5. Liu C. L. Introduction to Combinatorial Mathematics. New York: McGraw Hill, 1968. 393 p.

6. Dorfling M., Goddard W., Henning M.A., Mynhardt C.M. Construction of trees and graphs with equal dominating parameters. Discrete Math., 2006. Vol. 306, No. 21. P. 2647-2654. DOI: $10.1016 /$ j.disc.2006.04.031

7. Meddah N., Chellali M. Edges contained in all or in no minimum edge dominating set of a tree. Discrete Math. Algorithms Appl., 2019. Vol. 11, No. 4. Art. no. 1950040. DOI: 10.1142/S179383091950040X

8. Venkatakrishnan Y. B., Krishnakumari B. An improved upper bound of edge-vertex domination number of a tree. Inform. Process. Lett., 2018. Vol. 134. P. 14-17. DOI: 10.1016/j.ipl.2018.01.012 